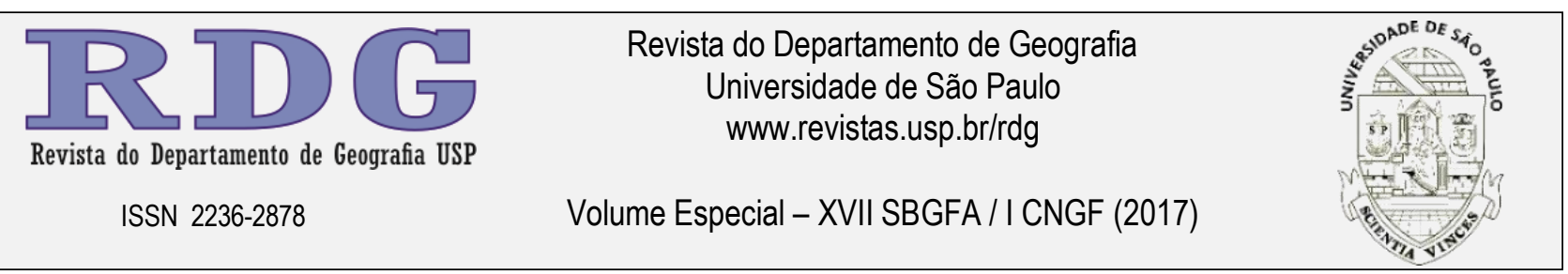

\title{
Vulnerabilidade Socioambiental das Áreas Sujeitas às Inundações no Município de São João Da Barra, RJ, Brasil
}

\author{
Socio-Environmental Vulnerability of Areas Subject to Flooding in the \\ Municipality of São João da Barra, RJ, Brazil
}

Talita de Oliveira Bracher Prates

Universidade Estadual de Campinas talitaprates@ige.unicamp.br

Raul Reis Amorim

Universidade Estadual de Campinas raul_reis@ige.unicamp.br

\begin{abstract}
Resumo: $O$ estilo de vida da sociedade atual, caracterizado pelo uso indiscriminado dos recursos naturais e pela intensa urbanização expõe as comunidades à diversos riscos e catástrofes naturais como, por exemplo, inundações e enchentes. O município de São João da Barra, localizada no estado do Rio de Janeiro, possui vários registros de acidentes ambientais e inundações que causaram prejuízos econômicos com deterioração de infraestruturas sociais abrangendo os setores da saúde da população. A falta de informação a respeito da vulnerabilidade da população exposta ao risco dificulta a elaboração de planos de resposta ou gerenciamento de risco eficazes na diminuição dos prejuízos causados pelas catástrofes ambientais. Este trabalho pretende contribuir com a formação de um banco de dados capaz de subsidiar a elaboração de políticas públicas e a tomada de decisão eficaz para minimização dos impactos negativos propondo o levantamento do perfil socioeconômico e a proposição de um índice de vulnerabilidade socioambiental capaz de indicar as áreas e populações com diferentes níveis de exposição ao risco, levando em consideração a suscetibilidade ambiental e as situações de pobreza e privação social da comunidade. Para tanto, foram utilizados os dados do censo demográfico de 2010 e a divisão territorial por setores censitários do IBGE no município de São João da Barra - RJ.
\end{abstract}

Palavras-chave: Vulnerabilidade socioambiental; Indicadores socioambientais; Risco ambiental; Pobreza.

\begin{abstract}
The lifestyle of modern society is characterized by indiscriminate use of natural resource and intense urbanization, which expose communities to risk of natural catastrophes such as inundation and flooding. The county of São João da Barra, located at the state of Rio de Janeiro, possesses many records of environmental accidents and flooding. These events caused economic deterioration of social infrastructure in different areas of its healthcare system. Lack of information regarding the vulnerability of exposed-to-risk population hampers the development of response plans and efficient risk management to reduce the losses caused by environmental catastrophes. This work aims to contribute with the formation of a database able to assist the elaboration of public policies and efficient decision making in order to minimize the negative impact of such tragedies. We proposed mapping the county's socioeconomic profile and the development of an index of socio-environmental vulnerability able to indicate the areas and populations with different levels of risk exposure taking into account the environmental susceptibility, degree of poverty, and social privation of the community. For doing so, we used data provided by the demographic census of 2010 and we considered territorial demarcation of São João da Barra in census sectors established by Brazilian Institute of Geography and Statistics (BIGS).
\end{abstract}

Keywords: Socio-environmental vulnerability; Socioenvironmental indicators; Environmental risk; Poverty. 


\section{INTRODUÇÃO}

A sociedade atual está exposta a riscos que são produzidos, em muitos casos, pelo uso indiscriminado dos recursos naturais. Somando-se o estilo de vida moderno com os efeitos das mudanças climáticas, da insuficiência de políticas para o gerenciamento de resíduos e com a crescente urbanização observados no último século, nota-se o aumento da intensidade e do número de vítimas dos acidentes ambientais.

Estudos recentes apontam que, na maioria das vezes, as áreas mais afetadas são habitadas por uma parcela da população de baixa renda e carente de infraestrutura básica (ALMEIDA, 2010; ALVES E TORRES, 2006; FREITAS E CUNHA, 2012). Esses fatos intensificam os riscos de desastres, diminuindo a capacidade de resposta e a qualidade de vida de tal parcela da população (CUTTER, 1996; FREITAS E CUNHA, 2012).

A falta de informação a respeito da vulnerabilidade da população exposta ao risco dificulta a elaboração de planos de resposta ou gerenciamento de risco eficaz na diminuição dos prejuízos causados pelas catástrofes ambientais.

A intenção do presente trabalho é fazer uma análise do município de São João da Barra - RJ, utilizando os resultados do Censo 2010, a partir de alguns conceitos e metodologias presentes no debate atual sobre população, espaço e meio ambiente. Neste sentido, o objetivo geral deste artigo é operacionalizar empiricamente o conceito de vulnerabilidade socioambiental, por meio da construção de um indicador, com integração de dados socioeconômicos e demográficos do Censo 2010 do IBGE e de dados que representem áreas de risco ambiental, especificamente as inundações.

O conceito de vulnerabilidade socioambiental é operacionalizado, aqui, a partir de uma definição que a descreve como a coexistência, cumulatividade ou sobreposição espacial de situações de pobreza e privação social e de situações de exposição a risco ambiental (ALVES, 2006). Para isso, construiu-se e analisou-se um índice de vulnerabilidade socioambiental, por meio de duas dimensões da vulnerabilidade - suscetibilidade e exposição ao risco ambiental -, combinando um indicador de renda domiciliar per capita com um indicador de exposição ao risco ambiental, tendo o setor censitário como unidade de análise. O indicador proposto neste artigo foi baseado e adaptado do trabalho de Alves (2013).

Com vistas ao exposto acima, este trabalho pretende contribuir com a formação de um banco de dados capaz de subsidiar a elaboração de políticas públicas e a tomada de decisão eficaz para minimização dos impactos negativos das inundações no município de São João da Barra, localizado no norte fluminense.

\section{BREVE DESCRIÇÃO DO MUNICÍPIO DE SÃO JOÃO DA BARRA}

Situado no note do estado do Rio de Janeiro, São João da Barra faz parte da microrregião de Campos dos Goytacazes. O município possui território de aproximadamente $458,611 \mathrm{~km}^{2}$. No que diz respeito à geomorfologia, esta região é conhecida como planície costeira do Rio Paraíba do Sul, composta por terrenos arenosos, terraços marinhos, campos de dunas e depósitos fluvio-lagunares, fazendo limites com os municípios de São Francisco do Itabapoana e Campos dos Goytacazes. São João da Barra é dividido em seis distritos (São João da Barra, Atafona, Grussaí, Barcelos e Pipeiras), no distrito de Atafona está presente a foz do Rio Paraíba do Sul (Figura 1). 


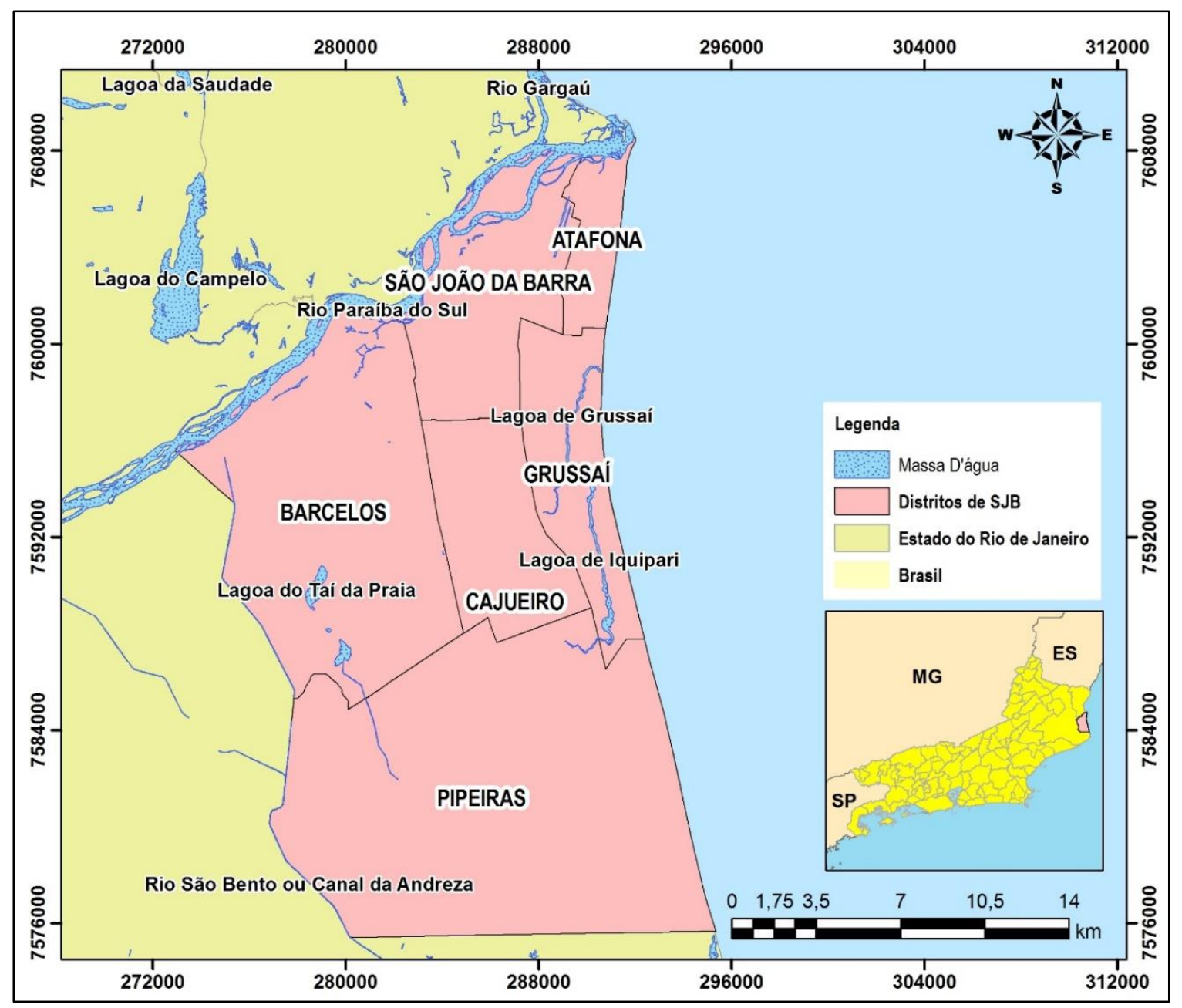

Figura 1: Localização e divisão territorial do município de São João da Barra - RJ

Segundo dados do Censo do IBGE (2010), São João da Barra possuía 32.747 habitantes, sendo $65,21 \%$ entre 15 e 59 anos, ou seja, população em idade ativa (PIA); o percentual de domicílios com rendimento per capita de até meio salário mínimo (abaixo da linha de pobreza) foi de 27,37\% e com rendimento per capta entre meio e um salário foi de $34,55 \%$.

Desde 2000 a região do norte fluminense experimenta mudanças no seu dinamismo econômico em virtude da exploração e produção de petróleo na bacia de Campos. O município de São João da Barra foi impactado por essa transformação com a instalação de um grande empreendimento portuário e industrial iniciado em 2007.

\section{REVISÃO SOBRE O CONCEITO DE VULNERABILIDADE}

Para entender o termo vulnerabilidade nas diversas abordagens científicas é preciso considerar, simultaneamente, o conceito de risco. Isso se deve ao fato de a vulnerabilidade aparecer no contexto dos estudos sobre risco em sua dimensão ambiental, num primeiro momento, e, mais tarde, no contexto socioeconômico (MARANDOLA E HOGAN, 2005).

A vulnerabilidade foi trazida para o debate ambiental no contexto dos estudos sobre os riscos principalmente pelo crescimento do interesse e preocupação com as populações em situações de risco. Profissionais passam a ocupar-se de estudos sobre enchentes e deslizamentos, entre outras situações em que o ambiente, conjugado a fatores socioeconômicos, expõe as populações a riscos, sobretudo nas cidades. 
O conceito de vulnerabilidade pode ser definido como uma situação em que estão presentes três elementos: exposição ao risco; incapacidade de reação; e dificuldade de adaptação diante da materialização do risco (MOSER, 1998). Outra linha de análise sobre vulnerabilidade tem origem nos estudos sobre desastres naturais (natural hazards) e avaliação de risco (risk assessment). Nesta perspectiva, a vulnerabilidade pode ser vista como a interação entre o risco existente em determinado lugar (hazard of place) e as características e o grau de exposição da população lá residente (CUTTER, 1994; 1996).

O conceito de vulnerabilidade não trata simplesmente da exposição aos riscos e perturbações, mas também da capacidade das pessoas de lidar com estes riscos e de se adaptar às novas circunstâncias. Nisto residem a importância e a inseparabilidade das dimensões social e ambiental da vulnerabilidade.

Nesse sentido, um conceito possível de ser usado para analisar estas questões é o de vulnerabilidade socioambiental, que pode ser definido como a coexistência, cumulatividade ou sobreposição espacial de situações de pobreza/privação social e de situações de exposição a risco e/ou degradação ambiental (ALVES, 2006).

\section{METODOLOGIA}

Como dito anteriormente, o objetivo geral deste artigo é operacionalizar o conceito de vulnerabilidade socioambiental, por meio da construção de um indicador, com integração de dados socioeconômicos e demográficos do Censo 2010 do IBGE e de dados que representem áreas de risco de inundações baseado e adaptado do indicador proposto por Alves (2013).

Em primeiro lugar estabeleceu-se duas dimensões (dimensão Ambiental e dimensão Socioeconômica) para a construção do indicador de vulnerabilidade socioambiental: A dimensão ambiental, baseada na exposição ao risco, neste caso, de inundações; e a dimensão socioeconômica e demográfica, baseada na renda domiciliar per capta. Ambas as dimensões tiveram como unidade de análise o setor censitário do Censo 2010 do IBGE.

Para a construção da dimensão ambiental do indicador utilizou-se cartas cartográficas da rede hidrográfica, a declividade, neste artigo, foi desconsiderada pois, o município de São João da Barra é predominantemente plano. A dimensão ambiental indica a porcentagem da área do setor censitário exposta ao risco ambiental.

Para tanto estipulou-se como área de risco de inundações uma margem de 50 metros de cursos d'agua, lagoas ou mar através da sobreposição espacial das cartografias das áreas de risco ambiental à malha digital dos setores censitários do Censo 2010 do IBGE para o município São João da Barra por meio de um Sistema de Informação Geográfica Em seguida, calculou-se o tamanho e a porcentagem da área de cada setor sobreposta às áreas de risco ambiental resultando na seguinte variável quantitativa contínua: porcentagem do território do setor censitário composto por áreas de risco ambiental. Finalmente, esta variável contínua foi convertida numa variável categórica com três categorias de risco ambiental: setores que possuem até 33\% da sua área exposta ao risco foram classificadas como áreas de baixo risco ambiental; setores de risco ambiental moderado são aqueles que possuem de 34 a $66 \%$ da sua área exposta ao risco e, finalmente, os setores considerados com alto risco ambiental são aqueles que possuem mais de $66 \%$ da sua área exposta ao risco (Figura 2).

Para operacionalizar o conceito de vulnerabilidade socioambiental, partiu-se de uma definição de Chambers (1989), que considera que a vulnerabilidade possui dois lados (ou duas dimensões): exposição ao risco; e suscetibilidade ao risco. Também foi utilizada uma definição operacional de vulnerabilidade socioambiental, que a descreve como a coexistência, cumulatividade ou sobreposição espacial de situações de pobreza e privação social e de situações de exposição a risco ambiental (ALVES, 2006).

Para a construção da dimensão socioeconômica do índice que, representa a suscetibilidade ao risco, foi empregado um indicador de renda domiciliar per capita, obtido nos resultados do Universo do Censo 2010 e convertido numa variável categórica ordinal com três categorias: indigentes para setores com renda domiciliar média per capita de até $1 / 2$ salário mínimo; pobre para setores com domiciliar média per capita de até dois salários mínimos e; não pobres com renda domiciliar média per capita superior a dois salários mínimos (Figura 2). 


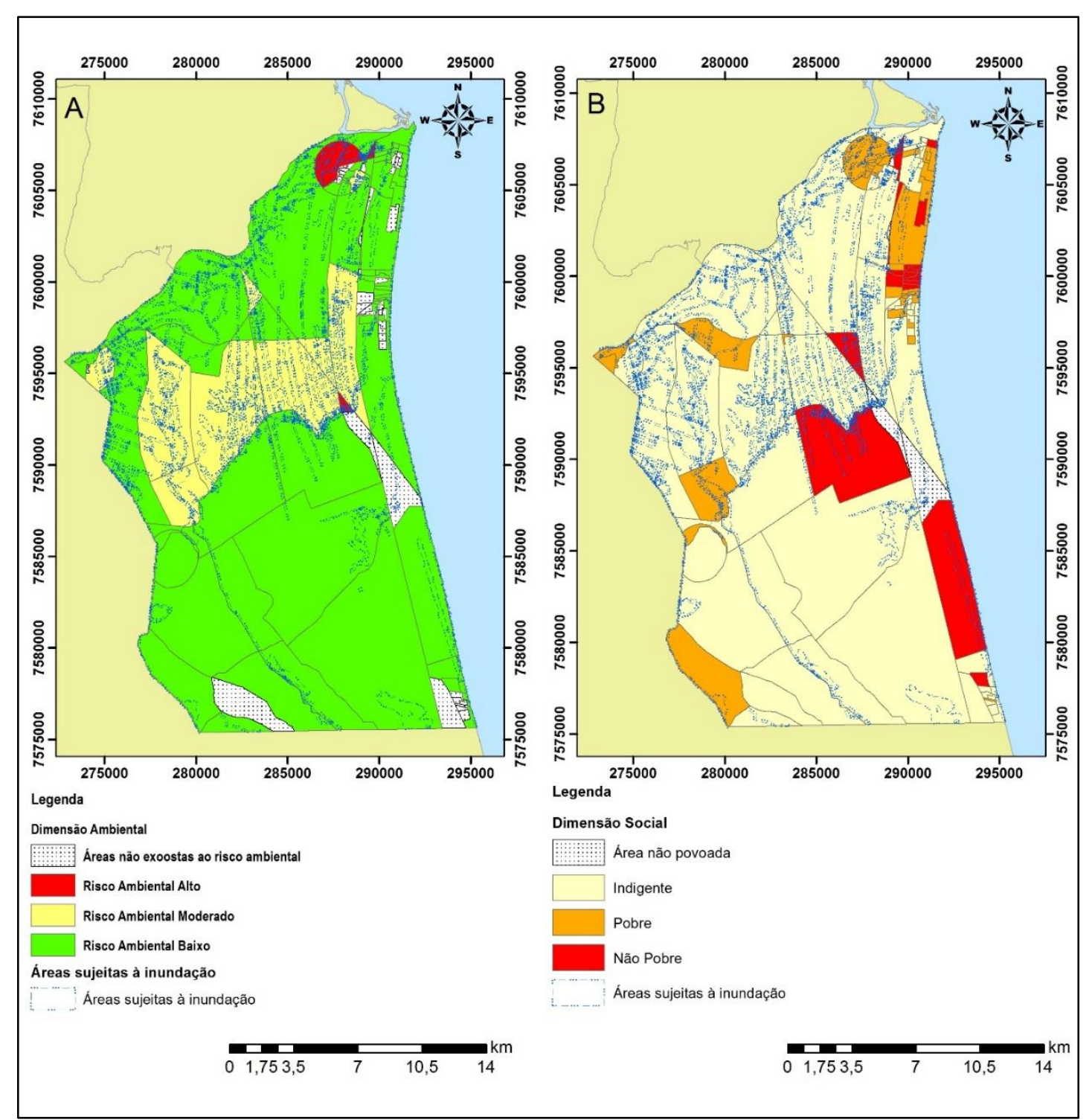

Figura 2: Sobreposição espacial das cartografias de risco ambiental e distribuição das categorias socioeconômicas à malha de setores censitários de São João da Barra -2010

Combinando as duas dimensões - suscetibilidade/pobreza e exposição ao risco ambiental -, gerou-se o índice de vulnerabilidade socioambiental, que é uma variável categórica ordinal com três categorias/grupos, descritos na Tabela 1.

Tabela 1: Construção do índice de vulnerabilidade socioambiental, por meio da combinação das dimensões suscetibilidade/pobreza e exposição ao risco ambiental

\begin{tabular}{|c|l|l|}
\hline \multicolumn{2}{|c|}{ Dimensões } & $\begin{array}{c}\text { Índice de Vulnerabilidade } \\
\text { Socioambiental }\end{array}$ \\
\hline Exposição ao Risco Ambiental & Socioeconômica/Suscetibilidade ao risco & Vulnerabilidade Alta \\
\hline \multirow{3}{*}{ Risco Ambiental Alto } & Indigente/Suscetibilidade Alta & Vulnerabilidade Alta \\
\cline { 2 - 3 } & Podre/Suscetibilidade Moderada & Vulnerada \\
\cline { 2 - 3 } & Não Pobre/Suscetibilidade Baixa & Vulnerabilidade Moderada \\
\hline \multirow{3}{*}{ Risco Ambiental Moderado } & Indigente/Suscetibilidade Alta & Vulnerabilidade Alta \\
\cline { 2 - 3 } & Podre/Suscetibilidade Moderada & Vulnerabilidade Alta \\
\cline { 2 - 3 } & Não Pobre/Suscetibilidade Baixa & Vulnerabilidade Baixa \\
\hline \multirow{3}{*}{ Risco Ambiental Baixo } & Indigente/Suscetibilidade Alta & Vulnerabilidade Moderada \\
\cline { 2 - 3 } & Podre/Suscetibilidade Moderada & Vulnerabilidade Baixa \\
\cline { 2 - 3 } & Não Pobre/Suscetibilidade Baixa & Vulnerabilidade Baixa \\
\hline
\end{tabular}


Importante ressaltar que ao se construir este índice de vulnerabilidade socioambiental, por meio de métodos de geoprocessamento e análise espacial, está sendo incorporado o espaço como uma dimensão analítica fundamental do presente estudo, e não apenas considerando o espaço uma mera representação dos dados censitários e ambientais (ALVES, 2013) (Figura 3).

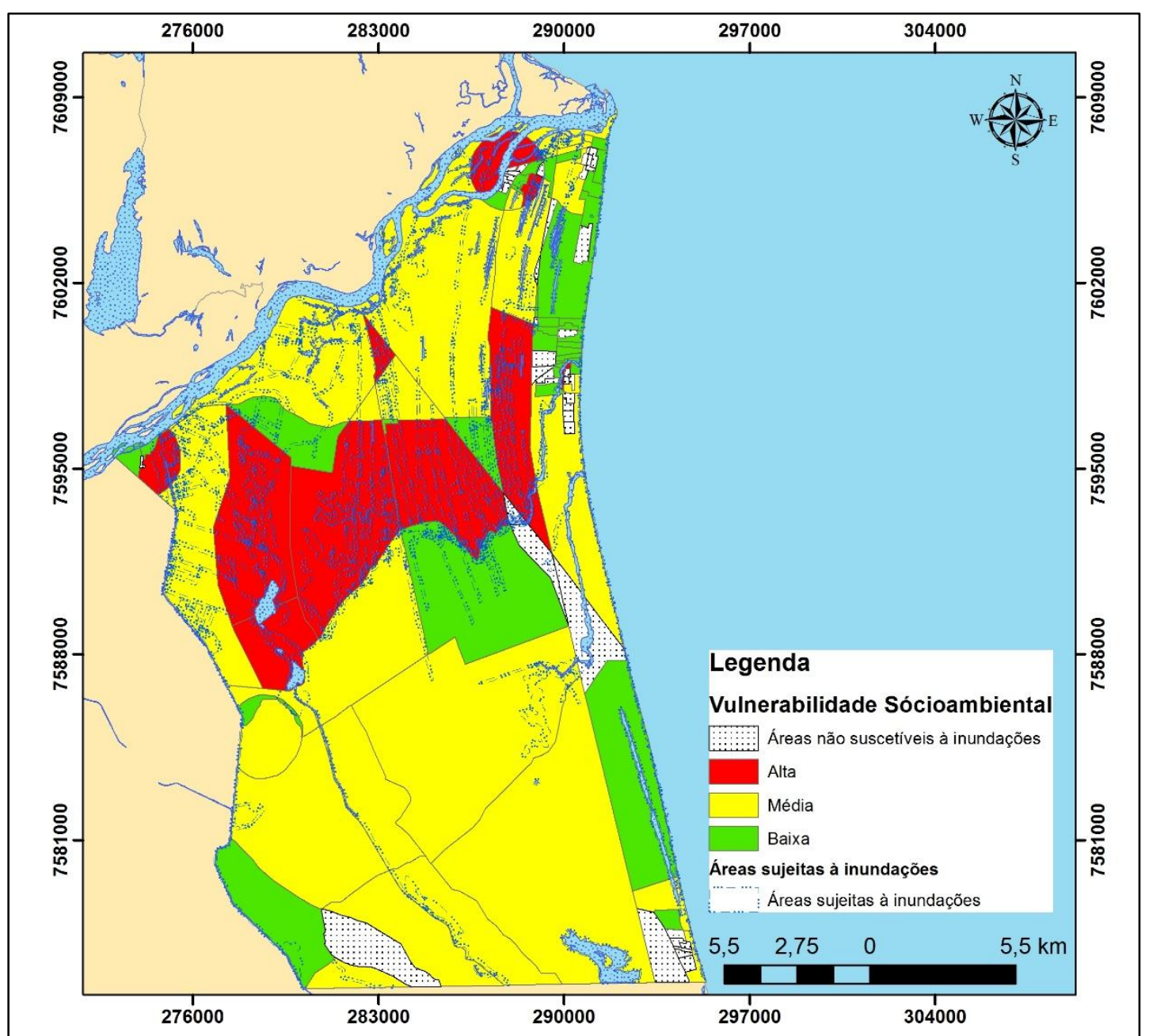

Figura 3: Classificação dos setores censitários, segundo grupos de vulnerabilidade socioambiental município São João da Barra - RJ

Classificando os setores censitários nos grupos de vulnerabilidade socioambiental, obtêm-se os seguintes volumes populacionais: nos setores que apresentaram alta vulnerabilidade socioambiental residem 3.747 pessoas correspondendo a $12 \%$ da população total do município; nos setores que apresentaram vulnerabilidade socioeconômica moderada residem 10.130 pessoas correspondendo a $31 \%$ da população total e nos setores classificados com baixa vulnerabilidade socioeconômica residem 7.936 pessoas correspondendo $24 \%$ da população total de São João da Barra. Esses resultados mostram que a menor faixa da população se encontra em situação de alta vulnerabilidade socioambiental.

Analisando comparativamente os indicadores socioeconômicos e demográficos entre os grupos de vulnerabilidade socioambiental com o objetivo de observar as expressivas diferenças entre eles e, assim, identificar e caracterizar as diversas combinações de situações de pobreza/ suscetibilidade e de exposição a risco ambiental no município de São João da Barra (Tabela 2), observou-se que a coleta de lixo está quase universalizada no município, já o abastecimento de água encontra-se em uma média de 50\% nos grupos de alta e moderada vulnerabilidade socioeconômica. Com relação à cobertura de esgoto nota-se a alarmante ineficiência em todo o município sendo representativa a utilização de fossas rudimentares, $80 \%$ dos domicílios que estão classificados com vulnerabilidade socioambiental moderada utilizam a fossa rudimentar e, 67\%dos domicílios de alta vulnerabilidade socioambiental. 
Não se percebe uma diferença expressiva na distribuição da renda entre as classes de vulnerabilidade socioambiental e não há representatividade expressiva de domicílios com renda per capita superior a dois salários mínimos (Tabela 2).

Tabela 2: Indicadores socioeconômicos e demográficos, por categoria de vulnerabilidade socioambiental

\begin{tabular}{|c|c|c|c|c|c|c|}
\hline \multirow{3}{*}{$\begin{array}{l}\text { Indicadores Socioeconômicos e Demográficos } \\
\text { População Residente }\end{array}$} & \multicolumn{6}{|c|}{ Vulnerabilidade Socioambiental } \\
\hline & \multicolumn{2}{|c|}{ Alta } & \multicolumn{2}{|c|}{ Moderada } & \multicolumn{2}{|c|}{ Baixa } \\
\hline & 3747 & $12 \%$ & 10130 & $31 \%$ & 7936 & $24 \%$ \\
\hline Número Total de Domicílios & 1184 & $11 \%$ & 3302 & $31 \%$ & 2591 & $24 \%$ \\
\hline Domić́lios com coleta de lixo & 1089 & $92 \%$ & 2939 & $89 \%$ & 2467 & $95 \%$ \\
\hline Domicílios com abastecimento geral de água & 698 & $59 \%$ & 1806 & $55 \%$ & 2145 & $83 \%$ \\
\hline Domicílios com abastecimento por poço de água & 472 & $40 \%$ & 1380 & $42 \%$ & 383 & $15 \%$ \\
\hline Domicílios com rede geral de esgoto & 347 & $29 \%$ & 328 & $10 \%$ & 632 & $24 \%$ \\
\hline Domicílios com fossa séptica & 22 & $2 \%$ & 216 & $7 \%$ & 638 & $25 \%$ \\
\hline Domicílios com fossa rudimentar & 792 & $67 \%$ & 2639 & $80 \%$ & 1261 & $49 \%$ \\
\hline Pessoas de cor branca & 2580 & $69 \%$ & 7305 & $72 \%$ & 4990 & $63 \%$ \\
\hline Pessoas de cor preta & 208 & $6 \%$ & 396 & $4 \%$ & 434 & $5 \%$ \\
\hline Domicílios com renda per capta de até $1 / 2$ salários mínimos & 446 & $38 \%$ & 1625 & $49 \%$ & 720 & $28 \%$ \\
\hline Domicílios com renda per capta de $1 / 2$ até 2 salários mínimos & 651 & $55 \%$ & 1534 & $46 \%$ & 1501 & $58 \%$ \\
\hline Domicílios com renda per capta superior a 2 salários mínimos & 91 & $8 \%$ & 147 & $4 \%$ & 343 & $13 \%$ \\
\hline
\end{tabular}

Em resumo, os resultados mostram que no município de São João da Barra os serviços básicos de saneamento são escassos em todo o território, demonstrando a necessidade de investimento em obras infraestrutura urbana. Também não há elevada diferença na distribuição da renda demonstrando que a vulnerabilidade não está diretamente associada a dimensões de pobreza e não há aglomerados subnormais na região, mas, a parcela da população com renda per capita acima de 2 salários mínimos exposta a alta vulnerabilidade socioambiental $(8 \%)$ possui maior resiliência ao dano. Por outro lado, apenas $12 \%$ da população residente no município apresentou vulnerabilidade alta, ou seja, o município apresenta possibilidades de elaboração de uma gestão quase individualizada para mitigação e prevenção dos possíveis prejuízos causados pelas enchentes e inundações.

\section{CONSIDERAÇÕES FINAIS}

Neste artigo, procurou-se operacionalizar empiricamente o conceito de vulnerabilidade socioambiental, por meio da construção de um índice de vulnerabilidade socioambiental, com integração de dados do Censo 2010 do IBGE e de dados de áreas de risco ambiental, para análise de situações de vulnerabilidade socioambiental em escala intraurbana no município São João da Barra - RJ.

Os resultados demonstraram uma necessidade de investimentos em infraestrutura urbana em todo o território do município e, por não apresentar aglomerados subnormais a vulnerabilidade não está ligada às dimensões de pobreza e, por outro lado, a situação de alta vulnerabilidade atinge somente 12\%da população total residente em São João da Barra, possibilitando a elaboração de gestão quase individualizada para mitigação e redução dos danos causados pelas enchentes e inundações. 
Assim, a construção de indicadores socioambientais, por meio de metodologias de geoprocessamento e análise espacial, possibilita identificar as áreas do município que necessitam de atenção e de gestão específica. Com isso, o presente trabalho traz uma importante contribuição metodológica às discussões já existentes no que tange a análise de vulnerabilidade socioambiental em escala intraurbana, ao realizar a integração de fontes censitárias de dados sociodemográficos com cartografias ambientais (áreas de risco). Cabe ressaltar que estas metodologias e indicadores podem ser replicados e adaptadas para outras áreas urbanas e metropolitanas do Brasil, uma vez que utilizam a malha digital de setores censitários do Censo 2010.

\section{REFERÊNCIAS BIBLIOGRÁFICAS}

ALMEIDA, L. Q. Vulnerabilidades Socioambientais de Rios Urbanos. Bacia Hidrográfica do rio Maranguapinho. 2010. 311p. (Doutorado em Geografia). Universidade Estadual Júlio de Mesquita Filho, Rio Claro. 2010.

ALVES, H. P. F.; TORRES, H. G. Vulnerabilidade socioambiental na cidade de São Paulo: uma análise de famílias e domicílios em situação de pobreza e risco ambiental. São Paulo em Perspectiva, v. 20, n. 1, p. 44-60, jan./mar. 2006.

ALVES, H. P F. Análise da vulnerabilidade socioambiental em Cubatão-SP por meio da integração de dados sociodemográficos e ambientais em escala intraurbana. Revista Brasileira de Estudos de População, v. 30, n. 22, p. 349-366/ jul./dez 2013.

CHAMBERS, R. Vulnerability, coping and policy. IDS Bulletin, v. 20, n. 2, 1989

CUTTER, S. L. Vulnerability to environmental hazards Progress in Human Geography, v. 20, n. 4, p. 529-539, 1996.

CUTTER, S. L. (org.). Environmental risks and hazards. London: Prentice-Hall, 1994.

FREITAS, M. I. C.; CUNHA, L. Modelagem de Dados Socioambientais Visando 78 Estudos de Vulnerabilidade: o Caso de 17 Concelhos do Centro de Portugal. Geonorte, v. 1, p. 816-829, 2012.

MARANDOLA JR., E.; HOGAN, D. J. Vulnerabilidade e riscos: entre geografia e demografia. Revista Brasileira de Estudos de População, v. 22, n. 1, p. 29-53, jan./jun. 2005.

MOSER, C. The asset vulnerability framework: reassessing urban poverty reduction strategies. World Development, v. 26, n. 1, p. 1-19, 1998. 